\title{
Localized electrons in dense heavy noble gases
}

\author{
S. Nazin and V. Shikin \\ Institute of Solid State Physics of Russian Academy of Sciences, Chernogolovka, Moscow District, 142432, Russia \\ E-mail: shikin@issp.ac.ru
}

Received January 4, 2009

\begin{abstract}
The paper addresses counterintuitive behavior of electrons injected into dense cryogenic media with negative scattering length $a_{0}$. Instead of expected polaronic effect (formation of density enhancement clusters) which should substantially reduce the electron mobility, an opposite picture is observed: with increasing $\left|a_{0}\right|$ (the trend taking place for inert gases with the growth of atomic number) and the medium density, the electrons remain practically free. An explanation of this behavior is provided based on consistent accounting for the non-linearity of electron interaction with the gaseous medium in the gas atom number density.
\end{abstract}

PACS: 71.10.-w Theories and models of many-electron systems.

Keywords: delocalized electron, heavy inert gases, negativ scattering length.

One of the most interesting and still important issues in physics of cryogenic media is the problem of electron clusters which emerged almost simultaneously with that of electron bubbles. However, it is much less transparent (compared to the case of electron bubbles) from the experimental side. Although there are some indications of the existence of electron clusters in xenon [1], they are not observed on the expected scale in media with comparatively high atomic polarizabilities (krypton, xenon) which are presumably likely to develop various electron autolocalization phenomena. On the contrary, the data on electron mobility in $\mathrm{Ar}, \mathrm{Kr}$, and $\mathrm{Xe}$ [2-4] reveal that electrons remain practically free (compared to mobility of positive ions possessing the structure of massive polaronic-type formations) in their motion, at least in the vicinity of the characteristic electron mobility peak which is observed for all heavy inert gases.

The existing description [5-7] of electron clusters in cryogenic media with negative scattering lengths $a_{0}$ employs the well-known approximation [8,9] for electron-medium interaction energy which is linear in the gas density $n$. Within this approximation, the minimal energy $V_{0}$ of delocalized electron injected into the gaseous media is calculated as

$$
V_{0}=\frac{2 \pi \hbar^{2} a_{0}}{m} n,
$$

where $m$ is the free electron mass. In terms of electron energy bands in solids, $V_{0}$ is the conduction band bottom energy. The case of $a_{0}>0$ corresponds to formation a single-electron bubble. On the other hand, a density enhancement domain with higher gas atom concentration (i.e., a cluster) may develop around the electron if $a_{0}<0$. The authors of Refs. 5-7 made every effort to provide a quantitatively accurate description of the gas density around the localized electron in the linear approximation. In addition to (1), they also introduced a non-local electron-gas interaction of the type

$$
E_{\mathrm{int}}=\iint d^{3} r^{\prime} d^{3} r n(r) v\left(r-r^{\prime}\right) \psi^{2}\left(r^{\prime}\right),
$$

where $\psi(r)$ is the electron wave function, took into account the deviation of the gas entropy contribution to the total free energy from the ideal gas, etc. Their final conclusions [5-7] practically coincide with the intuitively expected picture: the electron cluster should exist, and the electron localization degree as well as the cluster mass should monotonously grow with the density media and polarizability demonstrating exponential sensitivity to the temperature. The outlined approach [5-7] reveals no hints of electron mobility growth with the medium density [2-4].

In the present paper we show that in gaseous media with negative values of $a_{0}$ it is possible for electron, in a ceratin range of gas densities and temperatures, to form an autolocalized state involving formation of a cluster with the characteristic length $\lambda \gg a_{B}$ (where $a_{B}$ is the Bohr radius) if the electron-gas interaction $V_{0}(n)$ is treated beyond the linear approximation as in Eq. (1). The paper is organized as follows. First, we present the formal 
grounds for considering the non-linear behavior of $V_{0}(n)$. Then the electron cluster structure is calculated within the non-linear approach. Finally, the nature of deviations from non-linearity at small $n$ is discussed.

As already mentioned earlier, the existing theory of autolocalized electrons in cryogenic media employs the electron-gas interaction (1). However, the true minimal energy of an electron injected in inert gases with negative scattering length $(\mathrm{Ar}, \mathrm{Kr}, \mathrm{Xe})$ is substantially non-linear. Direct experiments [10] reveal that the energy $V_{0}(n)$ is only approximately linear at very small $n$ following Eq. (1). With further growth of $n$, the energy $V_{0}$ reaches some minimal value $V_{\min }$ at a certain density $n_{\min }$ after which it grows again (see Fig. 1; for $\operatorname{Ar} n_{\min }=13 \cdot 10^{21} \mathrm{~cm}^{-3,} V_{\min }=-0.3 \mathrm{eV}$, for $\mathrm{Kr} n_{\min }=14 \cdot 10^{21} \mathrm{~cm}^{-3}, V_{\min }=-0.66 \mathrm{eV}$, for Xe $n_{\min }=$ $=11 \cdot 10^{21} \mathrm{~cm}^{-3}, V_{\min }=-0.83 \mathrm{eV}$ ). Most important in our problem of cluster formation is the range of $n$ near $n_{\text {min }}$ where the derivative $\partial V_{0} / \partial n$ changes its sign. Indeed, it is natural to assume that dominating in the problem of self-consistent calculation of the gas atom number density $n(r)$ will be the densities minimizing the electron-gas interaction energy. Hopefully, the quantitative analysis can be based on any reasonable interpolation of the true $V_{0}(n)$ reproducing the correct minimum depth and position. In fact, we used the simplest polynomial approximation yielding in addition the correct slope of $V_{0}(n)$ at small $n$ :

$$
V_{0}(n)=\frac{2 \pi \hbar^{2} a_{0}}{m} n\left(1+A n+B n^{2}\right) .
$$

The parameters $A$ and $B$ were chosen for each inert gas in such a way that the correct values [10] of the minimum depth $V_{\min }$ and position $n_{\min }$ were reproduced.

Formally, the problem of finding the ground state of a single electron in the gaseous media reduces to minimizing the free energy $F$ of the entire «electron+gas» system with respect to variations of the (spherically symmetric)

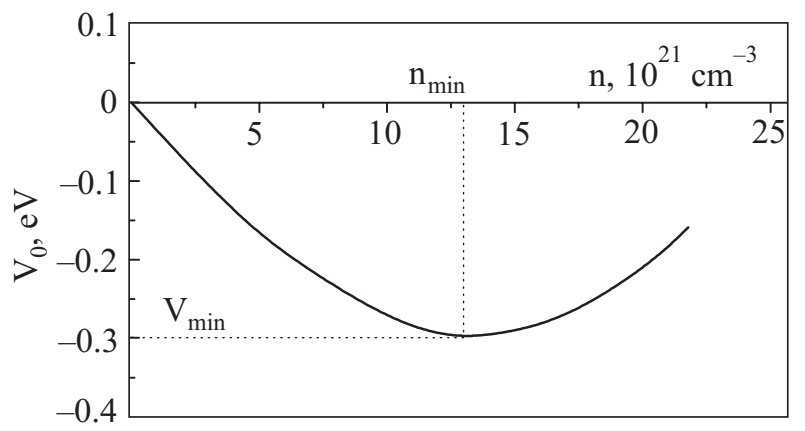

Fig. 1. Minimal energy of delocalized electron injected in argon as a function of gas atom number density as a typical example of behaviour of $V_{0}(n)$ for heavy inert gases with negative scattering length. electron wave function $\psi(r)$ and gas atom number density $n(r)[11,12]$,

$$
\begin{gathered}
F=\int d^{3} r \widetilde{F}(r), \quad \widetilde{F}=\frac{|\nabla \psi|^{2}}{2 m}+n T \ln (n B)+\widetilde{F}_{\text {int }}, \\
\widetilde{F}_{\text {int }}=V_{0}(n(r)) \psi^{2}(r),
\end{gathered}
$$

where $B(T)$ is a function of temperature which does not affect final results and therefore is not specified here. This procedure results in a set of two coupled equations for $n(r)$ and $\psi(r)$ :

$$
\begin{gathered}
-\frac{\hbar^{2}}{2 m} \nabla^{2} \psi+V(r) \psi=E \psi, \quad V(r)=V_{0}(n(r)), \\
4 \pi \int_{0}^{\infty} \psi^{2}(r) r^{2} d r=1, \\
n(r)=n_{g} \exp \left[-\frac{|\psi|^{2} \partial V_{0} / \partial n}{T}\right],
\end{gathered}
$$

where $n_{g}$ is the gas atom number density at infinity, $n(r)$ is the local atom number density, $\psi(r)$ is the electron wave function normalized to unity, and $T$ is the temperature.

To solve the Eqs. (5)-(7) we employed (just as in Refs. $11,12)$ the variational approach with $\psi(r)$ selected in the form

$$
\psi(r, k)=\left(\frac{k^{3}}{\pi}\right)^{1 / 2} \mathrm{e}^{-k r} .
$$

Here the variational parameter $k$ measures the electron localization. By substituting Eq. (8) into Eq. (7) and finding $n(r, k)$ one can calculate the free energy of the system $F(k)$ (4). To study the possibility of electron autolocalization at given $n_{g}$ and $T$ one should then plot the curve $\delta F(k)=F(k)-F_{\text {deloc }}$ (where $F_{\text {deloc }}=V_{0}\left(n_{g}\right)+$ $+N T \ln \left(n_{g} B(T)\right)$ is the total free energy of system consisting of a uniform gas and delocalized electron described by the wave function $\psi(r)=$ const, $N$ being the total number of gas atoms in the system and check if this curve has a minimum which is sufficiently deep compared to the temperature. For single electron bubbles, where $a_{0}>0$, this program was realized in $[11,12]$ where the linear approximation for $V_{0}$ was employed. The single electron bubble formation proves energetically favourable at sufficiently low temperatures and sufficiently high densities (threshold values of temperature and density follow the relation $n \sim T^{2 / 3}$ ), and all the parameters of arising bubble well satisfy the adopted assumptions: the bubble size is much larger than the interatomic distance, the free energy minimum depth substantially exceeds temperature, etc. We omit any quantitative details since for $a_{0}>0$ the non-linearity of $V_{0}(n)$ does not introduce any qualitative corrections to the bubble parameters and the resulting 
picture is practically identical to that obtained earlier $[11,12]$.

In the problem with $a_{0}<0$ we first mention that the relation

$$
n(r, k)=n_{g} \exp \left[+\frac{2 \pi \hbar^{2}\left|a_{0}\right||\psi(r, k)|^{2}}{m T}\right]
$$

following from Eqs. (7) and (1) leads in the linear theory to an unavoidable singularity in the density distribution $n(r \rightarrow 0)$ (in the adopted model which neglects inter-atomic repulsion at small distances nothing can prevent the arbitrarily strong shrinking of electron wave function and the corresponding growth of the gas density at the center of the cluster resulting in infinite reduction of the system free energy) as illustrated by variational calculations which yield for $\delta F(k)$ the results plotted in Fig. 2 (curve 1 ).

By employing a more general expression for $n(r, k)$ with $V_{0}(n)$ specified by Eq. (3) it is easy to see that the trend towards density enhancement around the localized electron taking place at relatively large distances from the cluster core and correctly described by Eq. (9) is stopped near the cluster center where the derivative $\partial V_{0} / \partial n$ changes its sign. It is also qualitatively clear that the halt in the density growth is actually important if the uniform gas density $n_{g}$ far from the cluster core is not too close to $n_{\text {min }}$. If the inequality $n_{g} \sim n_{\min }$ is satisfied, the cluster formation mechanism defined by Eqs. (5),(7) becomes inefficient (no energy gain can be acquired by tuning the gas density to its optimal value in the vicinity of the cluster center), and that is actually why electrons in heavy inert gases behave as practically free particles for gas densities close to $n_{\min }$.

Now that the singularity suppression mechanism is clear, one can apply the outlined variational procedure to quantitatively test the above qualitative picture concerning the possibility of electron cluster formation. Calculations reveal that at not too high temperatures $T$ there do

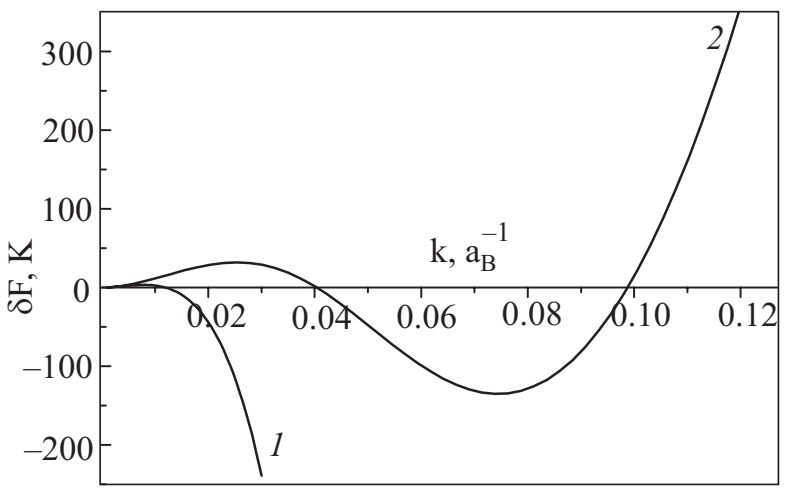

Fig. 2. Gain in the free energy due to electron localization in Xe as a function of the variational parameter $k$ in linear approximation (curve 1 ) and taking into account the non-linear behaviour of $V_{0}(n)$ (curve 2) calculated for $n_{g}=4 \cdot 10^{21} \mathrm{~cm}^{-3}, T=150 \mathrm{~K}$. exist density ranges where the free energy gain due to electron localization $\delta F(k)$ as a function of $k$ has a minimum with the depth exceeding $T$ (curve 2 in Fig. 2). Numerical results for Xe are shown in Fig. 3 where the free energy gain calculated for electron wave function defined by Eq. (8) and optimized with respect to $k$ is plotted. It is clearly seen that the localized state is only energetically favourable for not too low densities outside some interval around $n_{\min }$; the characteristic cluster radii prove to be (10-20) $a_{B}$. It should be noted that the structure of arising localized state proves to be completely different for $n_{g}<n_{\min }$ and $n_{g}>n_{\min }$. As already mentioned earlier, the free energy gain in the localized state occurs due to the proximity of the gas atom density around the electron $(r)$ to the density $n_{\min }$ providing the lowest possible interaction energy between the electron and the gas. Therefore, at $n_{g}<n_{\text {min }}$ the localized state corresponds to formation of a cluster (i.e. density enhancement in the vicinity of $r=0$ ), while at $n_{g}>n_{\text {min }}$ a single-electron bubble is formed with the gas atoms squeezed out of the vicinity of $r=0$ ).

Hence, the non-linear corrections to the interaction energy (1) behave in qualitatively different ways for $a_{0}>0$ and $a_{0}<0$. For positive scattering lengths, non-linear corrections to Eq. (1) only slightly modifies the overall picture of electron localization arising in the linear approach. On the contrary, for negative scattering lengths the presence of non-linearity in $V_{0}(n)$ becomes critically important since in the adopted model it is the only factor capable of preventing the cluster from shrinking to the Bohr length scale. Therefore, it is very desirable to study the deviation of $V_{0}(n)$ from the linear approximation (1), at least for small $n$. However, in spite of the fact that the problem of calculating $V_{0}(n)$ has been addressed in many works (e.g., see Refs. 13), currently available theoretical results are mainly numerical in nature and derived by replacing the disordered medium with imaginary crystalline

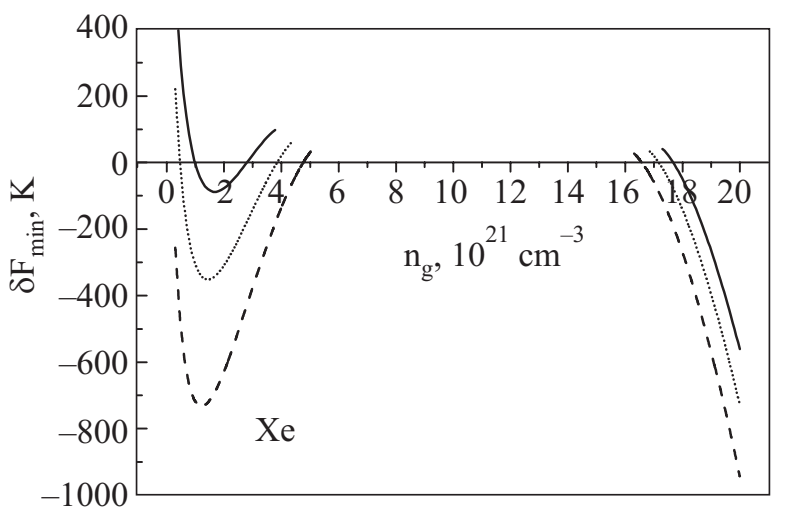

Fig. 3. Gain in free energy due to electron localization in Xe as a function of gas atom number density at three different temperatures, T, K: 150(----), $200(\cdots), 250$ (一). 
solid consisting of the gas atoms with appropriate density after which the conduction band bottom is calculated within the Wigner-Seitz model. Major efforts in these works have been concentrated on choosing the optimal pseudopotential describing the free electron interaction with the inert gas atom closed shell and correct screening of the long-range attracting potential $-\alpha e^{2} / 2 r^{4}$ due to the Coulomb interaction between the electron and polarizable gas atom, $\alpha$ being the atom polarizability. On the other hand, it is interesting to note that for short-ranged potentials the Wigner-Seitz model allows finding the asymptotic behaviour of $V_{0}(n)$ at small $n$ beyond the linear approximation through the scattering length $a_{0}$. Indeed, in that case the requirement of vanishing of the wave function first derivative at the spherical cell boundary (whose radius tends to infinity as $n \rightarrow 0$ ) results in the following expression for the conduction band bottom:

$$
V_{0}(n)=\frac{2 \pi \hbar^{2} a_{0} n}{m}\left(1+\frac{9}{5} \frac{a_{0}}{R}+O\left(\frac{a_{0}^{2}}{R^{2}}\right)\right),
$$

where $R=\left(\frac{3}{4 \pi n}\right)^{1 / 3}$. It is seen that the relative corrections to the linear approximation (1) are proportional to the small parameter $a_{0} / R<<1$ which is the prediction that can be tested experimentally. As an example, plotted in Fig. 4 are experimental data on low density behavior of $V_{0}(n)$ for argon in the $\left.\left(\left(V_{0}-V_{\operatorname{lin}}\right) / V_{\text {lin }}\right)^{3}, n\right)$ coordinates, where $V_{\text {lin }}=V_{0}$ from Eq. (1). It is obvious that $\delta V_{0}(n) / V_{\text {lin }} \propto a_{0} / R$, although the experimental proportionality coefficient is different from that predicted by Eq. (10). The reason for this discrepancy is most likely

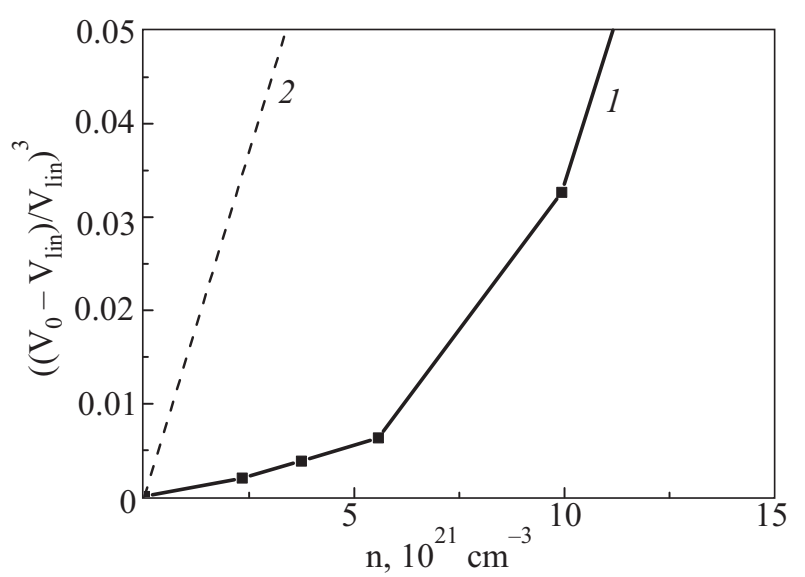

Fig. 4. Deviations from linear behaviour of $V_{0}(n)$ at small $n$ for Ar: experiment (1), 9/5 $a_{0} / R_{3}(2) ; V_{\text {lin }}=\frac{2 \pi \hbar^{2} a_{0}}{m} n$. the long-ranged nature of the effective potential for electron interaction with the gas atom containing the polarization contribution obeying the $r^{-4}$ law.

Thus, by taking into account the non-linear behaviour of $V_{0}(n)$, it is possible to extend the existing theory of electron autolocalization in dense gases with positive scattering lengths (single-electron bubbles in helium) to electrons in inert gases with negative scattering lengths and describe possible formation of electron clusters in these media. The clusters can arise at gas densities both lower and higher than $n_{\min }$ and are not formed at densities close to $n_{\min }$. The outlined picture is consistent with available data on electron mobility $\mu$ in dense cryogenic gases. The point is that with growing $n$ the possibility of interpreting the mobility $\mu$ in terms of single-particle collisions between the electrons and gas atoms is gradually lost. However, if under these conditions the electron still remains in an almost free non-localized state as suggested by the above analysis, it is natural to describe its interaction energy with the gaseous media responsible for scattering by the expression [14]

$$
\delta V_{0}=\frac{\partial V_{0}(n)}{\partial n} \delta n
$$

where $\delta n$ is the gas density fluctuation of the thermal origin. It is then obvious that the derivative $\partial V_{0}(n) / \partial n$ vanishing at $n=n_{\min }$ yields a peak in the density dependence of electron mobility. Hence, experimental observation of electron mobility peaks in all three heavy inert gases around the respective values of $n_{\min }$ can be considered as a confirmation of the absence of electron localization in the vicinity of the mobility peak.

This work was supported by Russian Foundation for Basic Research and the Program «Physics of Condensed Matter» of the Presidium of Russian Academy of Sciences.

1. S.S.-S. Huang and G.R. Freeman, J. Chem. Phys. 68, 1355 (1978).

2. L. Miller, S. Hove, and W. Spear, Phys. Rev. 166, 871 (1968).

3. L. Christophorou, R. Blaunstein, and D. Pittman, Chem. Phys. Lett. 18, 509 (1973).

4. Electronic Excitations in Liquefied Rare Gases, W.F. Schmidt and E. Illenberger (eds.), American Scientific Publishers, Stevenson Ranch, USA (2005).

5. A.G. Khrapak and I.T. Yakubov, Sov. Phys. JETP 42, 1036 (1975).

6. I. Yakubov and A. Khrapak, Chem. Phys. Lett. 39, 160 (1976).

7. I. Yakubov and A. Khrapak. Electrons in Dense Gases and Plasma, Nauka, Moscow (1981) (in Russian).

8. E. Fermi, Nuovo Cim. 11, 157 (1934). 
9. I.M. Lifshits, S.A. Gredeskul, and L.A. Pastur, Introduction to the Theory of Disordered Systems, Wiley-Interscience, New York (1988); S. Doniach and E.H. Sondheimer, Green's Functions for Solid State Physicists, Imperial College Press (1998).

10. I.T. Steinberger, Band Structure Parameters of Classical Rare Gas Liquids, in: Electronic Excitations in Liquefied Rare Gases, W.F. Schimidt and E. Illenberger (eds.), American Scientific Publishers, Stevenson Ranch, USA (2005), p. 51, ch. 3.
11. J.L. Levine and T.M. Sanders, Phys. Rev. 154, 138 (1967).

12. V.B. Shikin, Sov. Phys. Usp. 20, 226 (1977) (in Russian).

13. B. Springett, M. Cohen, and J. Jortner, Phys. Rev. 159, 183 (1967); I.T. Iakubov and V.V. Pogosov, Phys. Rev. B51, 14941 (1995); ibid. B53, 13362 (1996).

14. S. Basak and M.H. Cohen, Phys. Rev. B20, 3404 (1979). 